\title{
LIGHT RIPPLES AND THEIR EFFECTS PER PERSON AND PER BIRD
}

\author{
Dmitry Gladin ${ }^{1, *}$, and Alexey Kavtarashvili ${ }^{2}$ \\ ${ }^{1}$ LTD "TECHNOSVET GROUP", Russia \\ ${ }^{2}$ Laboratory of the Technology of Egg Production, Federal Scientific Center "All-Russian Research and Technological Institute of \\ Poultry" of Russian Academy of Sciences, Sergiev Posad, Russia
}

\begin{abstract}
The article discusses the influence of light pulsation on the physiological state of humans and birds, some aspects of its occurrence, the main characteristics and the alleged role in the deterioration of chickens' zootechnical indicators under the conditions of using light sources with light flux pulsations of different frequencies. The current state of lighting equipment in poultry farming is characterized by the rapid development of technologies and the active introduction of LED lighting. Having undeniable advantages, LED lighting systems have some technical features that are currently insufficiently studied and can affect the zootechnical indicators of poultry. A clear definition of the threshold values of the characteristics of the pulsation of illumination in the poultry house will make it possible to produce lighting equipment for poultry farming with a safe value of the pulsations of the luminous flux of light sources in their composition. The analysis of numerous literature sources has shown that the influence of light pulsation on the physiological state of humans and zootechnical indicators in birds is associated with a complex effect, depending on such characteristics as the depth and frequency of the pulsation of the light flux of light sources. Despite some differences in the values of the threshold impact of light pulsation, the nature of its impact is similar in humans and birds, which allows us to develop and apply targeted measures to reduce the impact of light pulsation on poultry and the deterioration of its zootechnical indicators.
\end{abstract}

\section{Introduction}

Currently, LED lighting is widely used in the Russian poultry industry, successfully replacing incandescent lamps and fluorescent light sources, having absolute advantages in improving the energy efficiency of lighting, improving the uniformity and creating the same light microclimate for the entire poultry population, as well as in choosing the color temperature (wavelength) of light sources [1-5]. However, the condition for effective control of the light flux and other characteristics of LED light sources is the use of pulsewidth modulation (PWM) of their supply voltage, which is characterized by a maximum ripple coefficient of $100 \%$ and a frequency of amplitude changes from several hundred $\mathrm{Hz}$ to several $\mathrm{kHz}$ [6]. For incandescent lamps and fluorescent light sources that are out of circulation but are still used in poultry houses, the ripple of the luminous flux reaches a ripple coefficient of $50 \%$ with a frequency from $100 \mathrm{~Hz}$ to several hundred $\mathrm{kHz}$ [7]. In the poultry houses where these light sources are used, the chicken and the person who maintains the equipment and ensures its vital activity are in the same light microclimate. Therefore, the influence of lighting characteristics, including light pulsation, on poultry enterprises should be considered not only from the point of view of zootechnical indicators of poultry, but also of employees who spend a large amount of time inside the poultry house.

The aim of this work was to generalize and analyze the current available scientific knowledge about the influence of light pulsation on birds and their characteristics, which lead to a deterioration in zootechnical indicators, and which can form a conceptual basis for our understanding of the mechanisms associated with this phenomenon.

The effect of light pulsation is best studied on a person, which is quite natural due to the accumulated knowledge about the structure of their own body, biological and physiological processes, as well as the ability to obtain information about the impact not only with the help of devices, but also directly from the subject.

The effect of light pulsation on poultry is much less studied, but the current development of poultry farming requires an intensive increase in poultry productivity, and such lighting characteristics are becoming an important element of the overall system of efficiency of poultry farms. The most rational way to determine the threshold values of the light pulsation parameters for a bird is to conduct studies based on its behavioral reactions at different values of flickering lighting, as well as on its direct impact on zootechnical indicators. The analysis of the obtained results should be carried out

\footnotetext{
* Corresponding author: gdv72.72@mail.ru
} 
in comparison with the well-known influence of this factor on humans, taking into account the peculiarities of the bird's visual system.

It is believed that the pulsation of illumination by a person can be consciously recorded up to a certain frequency, which is called the critical frequency of the merger of light flashes (KCHSSM). According to some works $[8,9]$, its value is in the range from 60 to $100 \mathrm{~Hz}$, and the specific value of the CCSSM is individual for each person. Light pulsations below this frequency can cause pronounced negative effects - malaise, impaired coordination and sleep, headaches, epileptic seizures, etc. Especially dangerous are the pulsation frequencies below $25 \mathrm{~Hz}$, which coincide with the alpha and theta rhythms of the human brain $[10,11]$.

Pulsations at frequencies higher than KCHSSM are not visible, are not fixed in consciousness, but can have a negative impact, the principle of which is of the following nature [12-14]:

1) When looking at objects, the eyes constantly make extremely small and very fast movements, called saccades (from an old French word translated as "the clap of a sail"). The pulsation of illumination leads to the fact that the saccades are lost, their number increases, a person sees an intermittent trail of phantoms of illuminated objects. This causes overexertion of the eye muscles and disrupts the normal functioning of the brain.

2) The light reflected from the object under consideration hits the retina, which sends electrical signals to the brain. If the light turns out to be pulsing, the spectrum of the generated signals changes, which disrupts the normal electrical activity of the brain. This causes fatigue and reduces concentration.

Light with high-frequency pulsations is detected by human visual receptors. At the same time, it is not processed as visual information, but directly affects the suprachiasmatic cells, the parventricular nuclei of the hypothalamus and the pineal gland. In turn, this directly affects a person's hormonal background, circadian rhythms, and related emotional well-being, performance, and fatigue [15].

The value of the light pulsation frequency, at which there are no negative consequences for a person, has different values in different studies. In one of the sources [16], the threshold is a frequency of $300 \mathrm{~Hz}$, above which any depth of illumination ripple is allowed, and the recommendation of the Institute of Electrical and Electronics Engineers IEEE PAR 1789 calculates the value of the critical frequency of illumination ripple (CEP) equal to $5.4 \mathrm{kHz}$, above which comfortable lighting for a person will be guaranteed.

There are studies that allow us to compare the reaction of a human and a chicken to the same effects of a light stimulus with different frequency and depth of flickering [17]. It is reported [18, 19] about the psychophysical operational study of the sensitivity of chickens to flickering, it is proposed to improve the model of human sensitivity, which allows it to be consistent with the data of observations of chicken. Also, for comparison, data on human sensitivity to flickering, collected under the same light stimulus as in chickens, are presented. In humans, the sensitivity to photopic flicker, as a function of its limiting modulation depth, shows a high-and low-frequency drop in the sensitivity level, reaching a maximum in the region of $10-15 \mathrm{~Hz}$ $[20,21]$. Two important processes that occur in the retina of the eye are included in the determination of sensitivity to light pulsation in humans. The first process, which performs the function of a low-pass filter, involves signal processing. During this processing, the photoreceptors act as the main elements. The second process, which acts as a high-pass filter, consists of inhibitory feedback, formed mainly from the connections of the horizontal cell and the amacrine cell in the inner and outer layers of the retina.

This inhibitory process is also responsible for the effect of brightness differences [22, 23]. The flicker sensitivity characteristic of each filter in a quantitative ratio can be described by the corresponding modulation transfer function (FPM). Early mathematical studies of flicker sensitivity demonstrate that FPM data is transmitted in cascades in order to reveal a person's perception of flicker over a huge range of different stimulus configurations. More recent studies of human sensitivity to flicker have led to a method of modeling FPM, including internal noise of sensorineural origin, the response to a signal detection filter located in the brain, as well as external noise of the stimulus itself.

In the course of the study, thirteen 8-month-old laying hens were placed under incandescent lamps in the conditions of natural day and night change (12T:12C) with illumination in the range from 5 to 50 lux. Before that, the chickens were raised under natural light in a special fenced area and in the open air. 12 volunteers were also selected, including women and men with normal vision aged 20 to 30 years. This age group was chosen in order to reduce the stated influence of age on the sensitivity to light pulsation [24].

The sensitivity of the bird to light flicker was determined in a range of stimulus brightness levels and compared directly with the level of human sensitivity to light flicker, measured under the same conditions. It was found that at five brightness levels $(10,100,200,500$ and 1000 candelas per square meter), the overall sensitivity of the bird to light pulsations is higher than that of a human, including high frequencies. The highest level of frequency tuning was found in the reaction of chickens. The critical CPSCM index in chickens (39.2, $54.0,54.0,57.4$, and $71.5 \mathrm{~Hz}$ ) was on average higher than the level of similar indicators in humans $(40.8,50.4$, $53.3,58.2$, and $57.4 \mathrm{~Hz}$ ) under conditions of increasing light stimulus brightness. The visual system of chickens demonstrates faster signal processing compared to humans.

Bird vision is different from human vision and is more developed in some aspects. On the one hand, humans have trichromatic color vision, which includes three types of photoreceptor cones in the retina with maximum absorption $(\lambda \max )$ at $420 \mathrm{~nm}, 530 \mathrm{~nm}$, and $560 \mathrm{~nm}$. They are commonly called cones that are sensitive to the blue, green and red parts of the spectrum. On the other hand, birds have four special types of single and double cones [25]. An additional type of single cones in the bird's retina, which is involved in color 
vision, makes the bird's vision tetrachromatic, that is, theoretically, it can distinguish twice as many colors as a human $[26,27]$. The fourth type of single cones can be sensitive to the ultraviolet and violet parts of the spectrum [28], and birds with each of these types of cones are classified as individuals sensitive to the ultraviolet [29] or to the violet part of the spectrum [30, 31 ], respectively. In both cases, sensitivity refers to short waves and allows birds to see in the region close to the ultraviolet spectrum, in the part that is invisible to humans. Birds use UV vision to make decisions about choosing a mate [32] and to search for food. In addition to the difference in color perception, birds showed the detection of flickering light at higher frequencies than in humans [33], and also showed a faster response to visual stimuli [34], due to a shorter pathway of the nervous system.

The interest in the sensitivity of the chicken to flickering light arises from a person's concern about the quality of life of poultry. Fluorescent lamps produce flickering light at 100 or $120 \mathrm{~Hz}$. If flickering is detected by a bird, it can create discomfort and increase stress. There are opposite results on the question of the bird's perception of lighting from a fluorescent lamp as flickering. In [35], we measured the critical frequency of flicker fusion and assumed that a flicker of $100 \mathrm{~Hz}$ is not perceived by a chicken, and in another source [36], the illumination and modulation coefficient of fluorescent light sources do not seem sufficient to perceive flicker, even at high brightness. The results obtained by extrapolating the function of the critical frequency of light pulsation fusion indicate that the chicken can perceive light from low-frequency fluorescent lamps as flickering. The results of the study [37] seem to be based on the conclusions that broilers exposed to lowfrequency light showed less physical activity.

Measurements of the critical light pulsation frequency in white laying hens were carried out using LED light of four different radiation spectra (white, white with the addition of the ultraviolet range (UV), yellow $(590 \mathrm{~nm})$ and only the ultraviolet range (UV) with a wavelength of $400 \mathrm{~nm}$ ), at four levels of light intensity [38]. The light intensity levels $(290,174,86$, and 43 candelas per $\mathrm{m} 2$ ) were adapted to the relative sensitivity of the domestic chicken eye cones to achieve equally active stimuli for different spectra. The results show significantly higher CCSSM values for white light with ultraviolet $(74,65,57$, and $47 \mathrm{~Hz})$ compared to just white light $(63,57,50$, and $45 \mathrm{~Hz})$, to relatively high critical light ripple frequencies $(76,70,60$, and $58 \mathrm{~Hz}$ ) for yellow and $(83,75,65$, and $55 \mathrm{~Hz})$ for pure UV light. The results for white light with ultraviolet compared to white light showed that, despite raising a chicken in artificial lighting conditions, its visual system responds better to the light conditions to which they were initially adapted in the external environment. In addition, there is reason to believe that the presence of UV light, used to find a partner and food, may also be an important condition for visual functions, such as sensitivity to light fluctuations.

\section{Conclusion}

Thus, the analysis of available literature sources shows that the impact of light pulsations on the bird in the conditions of artificial lighting of poultry houses is diverse and can affect its zootechnical indicators. Domestic chicken quite often acts as a model organism in various biomedical, physiological and behavioral experiments, and its visual system is sufficiently studied. Studies of determination the critical frequencies of light pulsation fusion for chickens are based on the analysis of the behavioral response of chickens and adults and make it possible to compare the results with the response to a similar effect for humans. The development of modern artificial lighting systems in poultry farming makes it possible to produce them with parameters that reduce the negative impact of light pulsations on the economy of the farm to a safe level.

\section{References}

1. Industrial poultry farming: a monograph; under the general editorship of V. I. Fisinin, Sergiev Posad, 531 (2016).

2. Adaptive resource-saving technology of egg production: monograph; under the general editorship of V. I. Fisinin and A. Sh. Kavtarashvili, Sergiev Posad, 351 (2016).

3. Progressive resource-saving technologies of egg production; under the general editorship of V. I. Fisinin, A. Sh. Kavtarashvili, Sergiev Posad, 167 (2009).

4. D. V. Gladin, LED local lighting in the production of chicken eggs: dis. ... Candidate of Agricultural Sciences, Sergiev Posad, 178 (2017).

5. Guidelines for the use of LED lighting in poultry farming; under the general editorship of A. Sh. Kavtarashvili and D. V. Gladin, Sergiev Posad, 171 (2020).

6. D. V. Gladin, A. Sh. Kavtarashvili, Control of LED lighting in a poultry house based on pulse-width modulation of the supply voltage, Poultry and poultry products, 4 , 52-56 (2020).

7. Reference book on lighting engineering; edited by Yu. B. Eisenberg and G. V. Boos, 4th ed. Pererab. and dop, Moscow, 892 (2019).

8. A. S. Sharakshane, S. V. Mamaev, R. S. Rotfullin, A.V. Porubov, Actual values of illumination pulsation created by modern light sources, Optical Journal, 84(1), 41-47 (2017).

9. I. S. Oshurkov, Grounded approach to the standards of the pulsations of led lighting, Modern electronics, 4, 68-71 (2013).

10. D. H. Kelly, Visual response to time-dependent stimuli amplitude sensitivity measurements, JOSA, 51, 422-429 (1961).

11. A. Wilkins, J. Veitch, B. Lehman, LED lighting flicker and potential health concerns: IEEE standard 
PAR1789update, Energy Conversion Congress and Exposition (ECCE), Atlanta: GA, 171-178 (2010).

12. A. Wilkins, Intermittent illumination from visual display units and fluorescent lighting affects movements of the eyes across text, Human Factors, 28, 75-81 (1986).

13. A. Kennedy, M. Brysbaert, W. S. Murray, The effects of intermittent illumination on avisual inspection taks The Quarterly J. Experimental Psychology, 51(1), 135-151 (1998).

14. A. L. Yarbus, Eye Movements and Vision, N. Y.: Plenum Press, 181 (1967).

15. V. A. Ilyanok, G. V. Samsonov, the pulsating Effect of light sources on the electrical activity of the human brain, Lighting, 5 (1963).

16. GOST R 54945-2012 Buildings and structures. Methods for measuring the light ripple coefficient, Moscow, Standartinform, 16 (2012).

17. J.R. Jarvis, N.R. Taylor, N.B. Prescott, I. Meeks, C.M. Wathes, Measuring and modelling the photopic flicker sensitivity of the chicken (Gallus g. domesticus). Vision Research 42(1), 99-106 (2002).

18. J. Rovamo, A. Raninen, S. Lukkarinen, K. Donner, Flicker sensitivity as a function of spectral density of external white temporal noise, Vision Research, 36, 3767-3774 (1996).

19. J. Rovamo, A. Raninen, K. Donner, The effects of temporal noise and retinal illuminance on foveal flicker sensitivity. Vision Research, 39, 533-550 (1999).

20. H. De Lange, Research into the dynamic nature of the human fovea cortex system with intermittent and modulated light. I. Attenuation characteristics with white and coloured light, Journal of the Optical Society of America, 48, 777-784 (1958).

21. D.H. Kelly, Theory of flicker and transient responses 1: uniform fields, Journal of the Optical Society of America, 61, 537-546 (1971a).

22. F. Ratliff, Mach bands: quantitative studies on neural networks in the retina, San Francisco: Holden-Day (1965).

23. F. Ratliff, B.W. Knight, J.I. Toyoda, H.K. Hartline, Enhancement of flicker by lateral inhibition. Science, 158, 392-393 (1967).

24. G.W. Brundrett, Human sensitivity to flicker, Lighting Research and Technology, 6, 127-143 (1974).

25. J.K. Bowmaker, L.A. Heath, S.E. Wilkie, D.M. Hunt, Visual pigments and oil droplets from six classes of photoreceptor in the retinas of birds, Vision Research 37, 2183-2194 (1997).

26. T.H. Goldsmith, Optimization, constraint and history in the evolution of eyes. The Quarterly Rewiev of Biology 65, 281-322 (1990).

27. D. Osorio, M. Vorobyev, C.D. Jones, Colour vision of domestic chicks, Journal of Experimental Biology 202, 2951-2959 (1999).
28. V.I. Govardovskii, L.V. Zueva, Visual pigments of chicken and pigeon. Vision Research 17, 537-543 (1977).

29. E.J. Maier, Spectral sensitivities including the ultraviolet of the passeriform bird Leiothrix lutea. Journal of Comparative Physiology, A 170, 709-714 (1992).

30. L.Y. Fager, R.S. Fager, Chicken blue and chicken violet, short wavelength sensitive visual pigments, Vision Research, 21, 581-586 (1980).

31. N.S. Hart, Vision in the peafowl (Aves: Pavo cristatus), Journal of Experimental Biology, 205, 3925-3935 (2002).

32. A.T.D. Bennet, I.C. Cuthill, J.C. Partridge, E.J. Maier, Ultraviolet vision and mate choice in zebra finches, Nature, 380, 433-435 (1996).

33. J.F.W. Nuboer, M.A.J.M. Coemans, J.J. Vos, Artificial lighting in poultry houses: do hens perceive the modulation of fluorescent lamps as flicker? British Poultry Science, 33, 123-133 (1992a).

34. N.B. Prescott, C.M. Wathes, J.R. Jarvis, Light, vision and the welfare of poultry, Animal Welfare, 12, 269-288 (2003).

35. J.L. Barbur, N.B. Prescott, R.H. Douglas, J.R. Jarvis, C.M. Wathes, A comparative study of stimulus-specific pupil responses in the domestic fowl (Gallus gallus domesticus) and the human, Vision Research, 42, 249-255 (2002).

36. J.R. Jarvis, N.R. Taylor, N.B. Prescott, I. Meeks, C.M. Wathes, Measuring and modelling the photopic flicker sensitivity of the chicken (Gallus g. domesticus), Vision Research, 42, 99-106 (2002).

37. F.M.G. Boshouwers, E. Nicaise, Responses of broiler chickens to high-frequency and lowfrequency fluorescent light, British Poultry Science, 33, 711-717 (1992).

38. D. Rubene, Functional Differences in Avian Colour Vision: A Behavioural Test of Critical Flicker Fusion Frequency (CFF) for Different Wavelengths and Light Intensities, Uppsala universite, 21, (2009). 